\title{
ISOLATED SINGULAR POINTS OF HARMONIC FUNCTIONS*
}

\author{
BY G. E. RAYNOR
}

Bôcher has given several theorems relating to the nature of a harmonic function in the neighborhood of an isolated singular point. $\dagger$ E. Picard $\ddagger$ has proved two of the theorems given by Bôcher in the paper mentioned above. In both papers the following fundamental theorem occurs.

If a function $f(x, y)$ is continuous and harmonic everywhere in the interior of a closed plane region with the exception of an isolated point $P$ in the neighborhood of which $f(x, y)$ tends to plus infinity for every mode of approach to $P, f$ is of the form $c \log (1 / r)+V$ in this neighborhood, where $c$ is a positive constant, $r$ the distance from $(x, y)$ to $P$ and $V$ is a function harmonic everywhere in the neighborhood of $P$, including $P$ itself.

The analogous theorem for three-space is also proved in each paper.

Picard's proof for the plane makes use of complex variables. However, as Bôcher points out, it is desirable to have an independent proof in order to follow out Riemann's idea of basing the theory of complex variables on the theory of real harmonic functions in two variables. Bôcher's discussion is general and applies to harmonic functions in any number of variables. However, both Bôcher and Picard, in the case of the theorem cited for three-space, apply Green's Formula to a region bounded partly by the surface

$$
f(x, y, z)=\text { constant }
$$

* Presented to the Society, October 31,1925 . See also a paper by O. D. Kellogg, On some theorems of Bôcher concerning isolated singular points of harmonic functions, which is to appear in the next issue of the Bulletin.

$\dagger$ This Bulletin, vol. 9 (1902-3), p. $455 \mathrm{ff}$.

‡ Bulletin De la Societe de France, vol. 52 (1924). 
Now this involves an integral over this surface and furthermore the integral contains the normal derivative of $f$ on the surface. In order to make the discussion complete it would seem that some consideration of the nature of the surface $f=c$ is necessary in order to know if $d f / d n$ has a meaning and if the integral exists. Such a discussion is of course possible but by no means simple. See, for example, the treatment of the analogous problem for Green's Function in the plane in Osgood's Funktionentheorie.* It is the purpose of the present paper to obtain for the plane additional results, as well as those of Bôcher, but by a different method. Furthermore, most of the present discussion can be generalized at once to three-space.

We suppose, then, that $f(x, y)$ is a function harmonic everywhere in a plane region $A$ except possibly at a single interior point $P$. We wish to study the nature of $f$ in the vicinity of $P$.

About $P$ as a center, and with radius $\gamma_{1}$, draw a circle $C_{1}$ which lies entirely in the region $A$. Also about $P$ draw a second circle $C_{2}$ with radius $r_{2}<r_{1}$. Let $V^{\prime}$ be the function which is harmonic everywhere in $C_{1}$ and which on $C_{1}$ coincides with $f$. This function we know exists and is given by Poisson's integral. In the following we shall indicate the region bounded by two concentric circles $C_{i}$ and $C_{j}$ by the symbol $C_{i} C_{j}$.

Now the difference

$$
f-V^{\prime} \equiv F
$$

is continuous and harmonic in $C_{1} C_{2}$ and is zero everywhere on $C_{1}$. Let $r$ be the distance of any interior point $(x, y)$ of $C_{1}$ to $P$. Then the function

$$
\log \frac{1}{r}-\log \frac{1}{r_{1}} \equiv W(r)
$$

is evidently also harmonic in $C_{1} C_{2}$ and takes the value

* First edition, p. 588; 2d ed., p. 673. 
zero on $C_{1}$. Since $V^{\prime}$ is given by Poisson's integral, we know that on $C_{1}, d V^{\prime} / d n$ exists and is continuous. Also since $f$ is harmonic on $C_{1}$ its normal derivative also exists and is continuous there. Hence the same is true of the difference $F \equiv f-V^{\prime}$. The same is seen directly to be true of $d W / d n$. Also, since both $W$ and $F$ are harmonic on $C_{2}$ their normal derivatives exist on this circle.

Let us now apply Green's Formula to the region bounded by $C_{1}$ and $C_{2}$. We have

$$
\int_{C_{1}}\left[F \frac{d W}{d n}-W \frac{d F}{d n}\right] d s=0,
$$

where the normal derivatives are taken toward the interior of the region $C_{1} C_{2}$. But on $C_{1}, F$ and $W$ are both zero, and hence

$$
\int_{C_{1}}\left[F \frac{d W}{d n}-W \frac{d F}{d n}\right] d s=0,
$$

or

$$
\int_{C_{2}} F \frac{d W}{d n} d s=\int_{C_{2}} W \frac{d F}{d n} d s
$$

But along $C_{2}$ we have $d W / d n=-1 / r$ which is constant on $C_{2}$ as is also $W$. Hence we have, substituting in (3),

$$
\int_{C_{2}} F d s=-r_{2} W\left(r_{2}\right) \int_{C_{2}} \frac{d F}{d n} d s .
$$

But by a well known theorem on harmonic functions

$$
\int_{C_{1} C_{2}} \frac{d F}{d n} d s=\int_{C_{1}} \frac{d F}{d n} d s+\int_{C_{1}} \frac{d F}{d n} d s=0,
$$

and hence (4) can be written

$$
\int_{C_{.}} F d s=r_{2} W\left(r_{2}\right) \int_{C_{1}} \frac{d F}{d n} d s .
$$


Let us set

$$
\frac{1}{2 \pi} \int_{C_{1}} \frac{d F}{d n} d s=c .
$$

We have then from (5) the result that the function $F$ satisfies the integral equation

$$
\int_{C_{2}} F d s=2 \pi r_{2}\left(\log \frac{1}{r_{2}}-\log \frac{1}{r_{1}}\right)
$$

where $C_{2}$ is any circle with $P$ as center, and radius $r_{2}<r_{1}$.

Now the general solution of (6) is easily seen to be

$$
F=c\left(\log \frac{1}{r_{2}}-\log \frac{1}{r_{1}}\right)+\Phi(x, y),
$$

where

$$
\int_{C_{2}} \Phi(x, y) d s=0
$$

and for our purposes $\Phi$ must be harmonic in $C$. Substituting in (1) we have

$$
\begin{aligned}
f & =c\left(\log \frac{1}{r}-\log \frac{1}{r_{1}}\right)+\Phi(x, y)+V^{\prime} \\
& =c \log \frac{1}{r}+\Phi(x, y)+V,
\end{aligned}
$$

where $V=V^{\prime}-c \log \left(1 / r_{1}\right)$. Since the form of $f$ is of course independent of any circle as $C_{2}$ the form (8) holds at any interior point of $C_{1}$ except $P$. Furthermore, on $C_{1}, \Phi \equiv 0$.

Point $P$ will in general be a singular point of $\Phi$, and we shall show as a matter of fact, unless $\Phi(x, y) \equiv 0$, that in the neighborhood of $P$ the function $\Phi$ will tend toward both plus infinity and minus infinity, that is, that there will exist a mode of approach to $P$ along which $\Phi$ will tend toward plus infinity and also a mode of approach to $P$ for which it will tend to minus infinity. Furthermore, the same statement will be seen to be true concerning the sum

$$
c \log \frac{1}{r}+\Phi \text {. }
$$


To prove this, let us write $\Phi$ in the form

$$
\Phi=\frac{1}{r} \Psi(x, y) .
$$

It is clear that in the open region bounded by $C_{1}$ and $P$ the function $\Psi(x, y)$ is continuous. We shall now define two functions $f_{1}(x, y)$ and $f_{2}(x, y)$ in this open region as follows:

$$
\begin{aligned}
& f_{1}(x, y)=\Psi(x, y) \text { at points where } \Psi(x, y) \geqq 0 \text {, } \\
& f_{1}(x, y)=0 \text { " " " } \Psi(x, y)<0 \text {, } \\
& f_{2}(x, y)=\Psi(x, y) \text { " } \quad \text { " } \quad \Psi(x, y) \leqq 0 \text {, } \\
& f_{2}(x, y)=0 \text { " } \quad \text { " } \quad \Psi(x, y)>0 \text {. }
\end{aligned}
$$

From the continuity of $\Psi(x, y)$ it is clear that $f_{1}$ and $f_{2}$ are continuous in their region of definition. We have thus

$$
\Psi(x, y)=f_{1}(x, y)+f_{2}(x, y) .
$$

From (7), since $1 / r$ is constant on any circle with $P$ as center we have

$$
\int_{C} \Psi(x, y) d s=0,
$$

where $C$ is such a circle interior to $C_{1}$. Hence

$$
\int_{C} f_{1}(x, y) d s+\int_{C} f_{2}(x, y) d s=0
$$

or in polar coordinates, with $P$ as pole, and any fixed line through $P$ as polar axis,

$$
\int_{0}^{2 \pi} f_{1} d \theta+\int_{0}^{2 \pi} f_{2} d \theta=0
$$

for any value of $r$. Consider now an arbitrary circle $C_{3}$ with $P$ as center and radius $r_{3}<r_{1}$. The expression*

$$
U(x, y)=\frac{1}{2 \pi r_{3}} \int_{C_{2}} \Phi d s-\frac{1}{\pi} \int_{C_{3}} \Phi \frac{\cos \phi}{d} d s
$$

* Goursat, Cours d'Analyse Mathématique, vol. 3, p. 222. 
defines a function $U$ which is harmonic at any point $(x, y)$ exterior to $C_{3}$ and is such that as the point $(x, y)$ approaches a point on $C_{3}, U(x, y)$ approaches the value of $\Phi$ at that point. In the above integrals $d$ is the distance from $(x, y)$ to a variable point $M$ on $C$, and $\phi$ the angle betwen $d$ and the radius of $C_{3}$ drawn to $M$.

By (7) the first of these integrals is zero, and hence

$$
U(x, y)=-\frac{1}{\pi} \int_{C_{3}} \Phi \frac{\cos \phi}{d} d s=-\frac{1}{\pi} \int_{0}^{2 \pi} \Psi \frac{\cos \phi}{d} d \theta .
$$

Substituting the value of $\Psi$ from (10) we obtain

$$
U(x, y)=-\frac{1}{\pi} \int_{0}^{2 \pi} f_{1} \frac{\cos \phi}{d} d \theta-\frac{1}{\pi} \int_{0}^{2 \pi} f_{2} \frac{\cos \phi}{d} d \theta .
$$

But $f_{1}$ and $f_{2}$ are of constant sign and hence applying the first theorem of the mean for integrals to (14) we have

$$
\begin{aligned}
U(x, y)= & -\frac{1}{\pi}\left\{\left[m_{1} \frac{\cos \phi}{d}\right] \int_{0}^{2 \pi} f_{1} d \theta\right. \\
& \left.+\left[m_{2} \frac{\cos \phi}{d}\right] \int_{0}^{2 \pi} f_{2} d \theta\right\}
\end{aligned}
$$

where $[m \cos (\phi / d)]$ indicates a mean of $\cos (\phi / d)$. But by (12), (15) becomes

$$
U(x, y)=-\frac{1}{\pi}\left\{\left[m_{1} \frac{\cos \phi}{d}\right]-\left[m_{2} \frac{\cos \phi}{d}\right]\right\} \int_{0}^{2 \pi} f_{1} d \theta .
$$

Let $\bar{f}_{1}\left(r_{3}, \theta\right)$ indicate the maximum value of $f_{1}$ on $C_{3}$. Then

$$
|U(x, y)| \leqq 2\left|\left[m_{1} \frac{\cos \phi}{d}\right]-\left[m_{2} \frac{\cos \phi}{d}\right]\right| \bar{f}_{1}\left(r_{3}, \theta\right) .
$$

Suppose now that $\bar{f}_{1}$ approaches zero as $r_{3}$ approaches zero. Then, since for any point on $C_{1},[m \cos (\phi / d)]$ is bounded, $|U(x, y)|$ can be made less than $\epsilon / 4$ at all points on $C_{1}$ by taking $r_{3}$ sufficiently small, $r_{3}=r_{3}^{\prime}$, say. Now on $C_{3}, \Phi$ and $U$ are equal, while on $C_{1}, \Phi=0$. Hence in the region $C_{1} C_{3}$, 
$\Phi$ will differ from $U$ by less than $\epsilon / 2$. Now consider any point $(a, b)$ except $P$, interior to $C_{1}$. We can take $r_{3}$ so small that $(a, b)$ lies in $C_{1} C_{3}$. Also by (16), $r_{3}$ can be taken so small, $r_{3}=r_{3}^{\prime \prime}$ say, that $U(a, b)<\epsilon / 2$. Hence by taking $r_{3}$ equal to the smaller of $r_{3}^{\prime}$ and $r_{3}^{\prime \prime}$ we have

$$
|\Psi(a, b)|<\epsilon \text {. }
$$

Since $\epsilon$ is any arbitrarily small number and $(a, b)$ is any point in $C_{1}$ except $P$, we have the result that if $\bar{f}_{1}$ approaches zero as $r_{3}$ approaches zero, $\Psi$ is identically zero in $C_{1}$.

Therefore, unless $\Phi \equiv 0$, there must be a mode of approach to $P$ for which $f_{1}$ remains greater than some positive constant $c_{1}$, or in other words for which $\Phi$ remains greater than $c_{1} / r$. In the same manner we can prove that there exists a mode of approach to $P$ for which $\Phi$ remains less than $-c_{2} / r$ where $c_{2}$ is a positive constant.

Suppose the constant $c$ in (8) is positive. It is easy to prove that

$$
\lim _{r \rightarrow 0}\left[c \log \frac{1}{r}-\frac{c_{2}}{r}\right]=-\infty
$$

and hence for some mode of approach to $P$

$$
\lim _{r \rightarrow 0}\left[c \log \frac{1}{r}+\Phi(x, y)\right]=-\infty .
$$

In the same way, if $c$ is negative, for some mode of approach to $P$

$$
\lim _{r \rightarrow 0}\left[c \log \frac{1}{r}+\Phi(x, y)\right]=+\infty .
$$

We can now formulate the following theorems.

THEOREM I. In the neighborhood of an isolated singular point $P$, a harmonic function $f(x, y)$ is of the form

$$
f(x, y)=c \log \frac{1}{r}+\Phi(x, y)+V(x, y)
$$


where $c$ is a constant, $r$ the distance from $(x, y)$ to $P, \Phi(x, y)$, unless it is identically zero, harmonic in the neighorhood of $P$ and such that there exist modes of approach to $P$ for which the sum $c \log (1 / r)+\Phi$ tends towards plus infinity and also toward minus infinity; and $V$ is harmonic everywhere in the neighborhood of $P$, including $P$.

THEOREM II. If a harmonic function $f(x, y)$ is bounded in the deleted neighborhood of an isolated singular point, it is in this neighborhood equal to a function which is harmonic in this neighborhood and also at $P$, and the singularity can be removed by defining $f(P)$ to be equal to $\lim f(x, y)$ as $(x, y)$ approaches $P$.

For the constant $c$ and the function $\Phi$ must be identically equal to zero if $f$ is bounded and we have $f=V$ in the neighborhood of $P$.

THEOREM III. If a harmonic function tends toward plus infinity (minus infinity) in the neighborhood of an isolated singular point, it is of the form $f=c \log (1 / r)+V$ where $c$ is a positive (negative) constant and $V$ is harmonic everywhere in this neighborhood, including $P$.

For by Theorem I, $\Phi$ must be identically zero in this case.

THEOREM IV. A harmonic function $f(x, y)$ cannot have an isolated singular point $P$ which is such that for certain modes of approach to $P, f(x, y)$ tends toward plus infinity (minus infinity) and remains finite for all other modes of approach to $P$.

For if $\Phi(x, y) \equiv 0$, we have the case described in Theorem III, while if $\Phi(x, y)$ is not identically zero, Theorem IV follows from Theorem I.

WESI EYAN UNIVERSITY 